\title{
p-Type Doping of Epitaxial Graphene by p-tert-Butylcalix[4]arene
}

\author{
Sunmin Park, Sena Yang, Ki-jeong Kim, Kwanghyun No, and Hangil Lee ${ }^{*}$ \\ Department of Chemistry, Sookmyung Women's University, Seoul 140-742, Korea. *E-mail: easyscan@sookmyung.ac.kr \\ ${ }^{\dagger}$ Pohang Accelerator Laboratory, POSTECH, Pohang 790-784, Korea \\ Received July 26, 2010, Accepted August 15, 2010
}

\begin{abstract}
The Chemical Doping of epitaxial graphene (EG) due to $p$-tert-butylcalix[4]arene was investigated using high resolution photoemission spectroscopy (HRPES). The measured work function changes verified that increased adsorption of the $p$-tert-butylcalix[4]arene on EG showed $p$-type doping characteristics due to charge transfer from the graphene to the $p$-tert-butylcalix[4]arene through the hydroxyl group. A single oxygen bonding feature associated with the $\mathrm{O} 1 s$ peak was clearly observed in the core-level spectra, indicating the presence of one equivalent adsorption state.
\end{abstract}

Key Words: p-tert-Butylcalix[4]arene, Chemical doping, Epitaxial graphene, HRPES

\section{Introduction}

Graphene, which is formed by a single layer of graphite with two-dimensional $s p^{2}$ orbital, has recently attracted considerable attention due to its remarkable electronic and optical properties. ${ }^{1}$ Its versatile applications in many emerging areas, such as graphene-based electronic devices, have inspired many research groups to actively study the material's properties. It may be possible to manipulate both the occupation of electronic states near the Fermi level and the magnitude of the gap between the valence band and the conduction band by controlling the carrier density using metals or molecules in the graphene layer. ${ }^{2-7}$ Functionalizing the graphene surfaces using a direct chemical grafting process is an important method for further expanding the material's potential. ${ }^{8}$ Several schemes have been proposed to modulate the band gap in graphene, ${ }^{9-13}$ but relatively few of these schemes have been actualized experimentally. One heavily debated example is the introduction of substrate-induced gaps in graphene supported on $\mathrm{SiC}$ substrates. ${ }^{14,15}$ Hence, application of graphene to carbon-based electronic devices requires the doping of molecules and metals on the graphene layers such that the carrier density of the graphene layer is controlled. ${ }^{1,3,16}$

In this work, we doped epitaxial graphene (EG) with $p$-tertbutylcalix[4]arene. $p$-tert-Butylcalix[4]arene is characterized by a three-dimensional basket shape in which four hydroxyl groups interact via hydrogen bonding to stabilize the concave conformation. p-tert-Butylcalix[4]arene can also accelerate reactions inside its cavity through a combination of local concentration effects and polar stabilization of a transition state. Hence, we predicted that $p$-tert-butylcalix[4]arene could successfully functionalize the graphene surfaces because it includes hydroxyl groups that may be used to facilitate chemical doping. Increasing the deposition of $p$-tert-butylcalix[4]arene was expected to produce doping and to modulate the EG surface properties.

Here, we characterized the electronic structure of $p$-tertbutylcalix[4]arene adsorbed on EG as a function of the work function and core levels, measured by high resolution photoemission spectroscopy (HRPES).

\section{Experimental Methods}

p-tert-Butylcalix[4]arene, shown in Fig. 1, is synthesized by the condensation of $p$-tert-butylphenol with formaldehyde and sodium hydroxide. ${ }^{17}$ HRPES experiments were performed under ultrahigh vacuum conditions (base pressure $=1.5 \times 10^{-10}$ Torr) at the 8A2 beamline of the Pohang Accelerator Laboratory (PAL), which is equipped with an electron analyzer (SES100, Gamma Data Scienta). The nitrogen-doped $\left(\mathrm{N}_{\mathrm{D}} \sim 9 \times 10^{17} \mathrm{~cm}^{-3}\right)$ Si-terminated 6H-SiC(0001) substrate was purchased from Cree Research (USA). The annealing temperature was monitored with an infrared pyrometer assuming an emissivity of 0.90 . After degassing the substrate to $900{ }^{\circ} \mathrm{C}$ for $24 \mathrm{~h}$, we deposited silicon $(1 \AA / \mathrm{min})$ for 5 minutes at $900{ }^{\circ} \mathrm{C}$ to produce a Si-rich surface. Finally, the substrate was graphitized at temperatures up to $1180^{\circ} \mathrm{C}$ (for two minutes) to produce monolayer graphene.

p-tert-Butylcalix[4]arene was purified by turbo pumping to remove impurities before dosing on the EG surface. A direct solid doser was used to introduce the $p$-tert-butylcalix[4] arene. The $\mathrm{C} 1 s, \mathrm{O} 1 s$ core-level spectra and valence spectra were measured using photon energies of 320,600 , and $130 \mathrm{eV}$. Secondary electron emission spectra ( $-20 \mathrm{~V}$ sample bias) were measured at a photon energy of $80 \mathrm{eV}$. The binding energies of the core-level spectra were determined with respect to the binding energies of the clean $\mathrm{Au} 4 f$ core level and the valence band (Fermi energy) for the same photon energy. All spectra were recorded in the normal emission mode. The photoemission spectra were carefully analyzed using a standard nonlinear least-

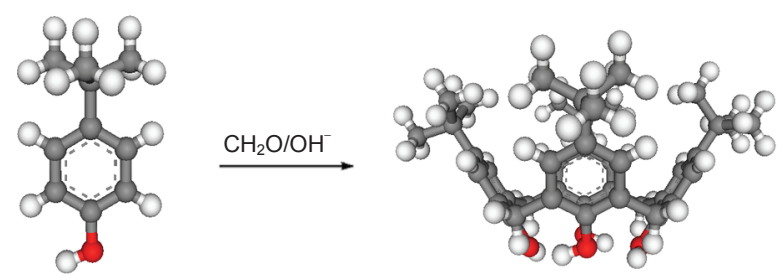

Figure 1. Synthesis of $p$-tert-butylcalix[4]arene. Each red, gray, and white ball indicates oxygen, carbon, and hydrogen, respectively. 
squares fitting procedure with Voigt functions. ${ }^{18}$

\section{Results and Discussion}

First, we carried out core-level HRPES experiments to characterize the structure of $p$-tert-butylcalix[4]arene adsorbed on EG. Figure 2(a) shows the $\mathrm{C} 1 s$ core-level spectrum of a graphene layer grown on $6 \mathrm{H}-\mathrm{SiC}(0001)$ obtained after annealing at $1180{ }^{\circ} \mathrm{C}$ for 2 minutes. At this annealing temperature, the thickness of the graphene layer was almost $1.0 \mathrm{~mL}$ (monolayer), as confirmed by PES (attenuation of the Si $2 p$ signal, data not shown) and low-energy electron diffraction (LEED) experiments. ${ }^{19}$ As shown in Fig. 2(a), three distinct peaks emerged at 285.5, 284.8, and $283.6 \mathrm{eV}$, which were assigned, respectively, to $s p^{2}$ carbon atoms in the interface layer weakly interacting with the carbon atoms of the underlying substrate (marked as S2), carbon atoms in the interface layer bound to one Si and three $\mathrm{C}$ atoms in the underlying substrate (marked as $\mathrm{S} 1$ ), and $\mathrm{SiC}$ (marked as $\mathrm{SiC}$ ). ${ }^{20,21}$ After confirming the pristine state of the monolayer graphene by $\mathrm{C} 1 s$ core-level spectroscopy, we exposed the EG to $4000 \mathrm{~L}$ p-tert-butylcalix[4]arene. Figure 2(b) shows the $\mathrm{C} 1 s$ core-level spectrum after the adsorption of $p$ tert-butylcalix[4]arene (4000 L) on EG, during which the substrate temperature was maintained at $100{ }^{\circ} \mathrm{C}$ to enhance the adsorption rate. In this spectrum, we observed a decrease in the intensity of the $\mathrm{S} 2$ peak $(\sim 18 \%$, see the inset of the left panel; Fig. 2(a), (b)) induced by the interfacial carbon. Because S2 included several defects, a decrease in this peak intensity due to $p$-tert-butylcalix[4] arene exposure was reasonable. Excluding this phenomenon, we found no changes in the $\mathrm{C} 1 \mathrm{~s}$ spectrum. Next, we focused on the presence and bonding features of the oxygen atoms in the $p$-tert-butylcalix[4]arene. We acquired $\mathrm{O} 1 s$ core-level spectra to track the adsorption of $p$-tertbutylcalix[4]arene on EG. The O $1 s$ core-level spectrum of the

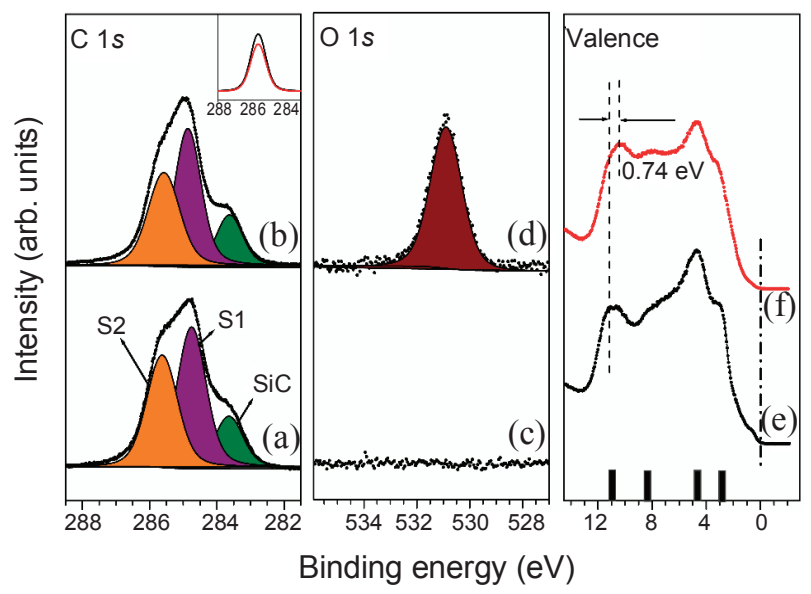

Figure 2. (Left panel) $\mathrm{C} 1 s$ core-level spectra obtained at photon energy of $330 \mathrm{eV}$. The inset of the left panel indicates the $\mathrm{S} 2$ intensity difference between EG (black colored spectrum) and a 4000L (red colored spectrum)deposition of $p$-tert-butylcalix[4] arene on EG. (Middle panel) $\mathrm{O} 1 \mathrm{~s}$ core-level spectra obtained at photon energy of $600 \mathrm{eV}$. (Right panel) Valence band spectra obtained at photon energy of $130 \mathrm{eV}$. The inset of the right panel shows a magnification of the valence-band spectra of the Fermi level. (a), (c), and (e) EG and (b), (d), and (f) $4000 \mathrm{~L}$ p-tert-butylcalix[4] arene adsorbed on EG at $100{ }^{\circ} \mathrm{C}$. system with $p$-tert-butylcalix[4] arene adsorbed on EG (Fig. 2(d)) displayed a single peak at $532.8 \mathrm{eV}$, which was assigned to oxygen interacting with the EG. Hence, through spectral analysis, we clearly confirmed that p-tert-Butylcalix[4]arene adsorbed on EG without bond breaking.

Finally, we acquired angle-integrated valence band spectra to clarify the molecular doping effects of $p$-tert-butylcalix[4] arene adsorption. Figure 2(e) shows four distinct hybridized features that evolved at 3.0, 4.7, 8.2, and $11.0 \mathrm{eV}$ below $E_{\mathrm{F}}$, which agree well with the results obtained by Johansson et al. at $1150{ }^{\circ} \mathrm{C} .{ }^{22,23}$ The strong peak near $4.7 \mathrm{eV}$ was assigned to the strong $\pi$ band at the $\mathrm{M}$ point. ${ }^{24}$ Figure 2(f) shows a remarkable change in the electronic structure after deposition of $p$-tertbutylcalix[4]arene (4000 L). First, we observed that the peak was marked by a shift to a lower binding energy. Compared to the graphene layered surface (Fig. 2(e)), the binding energy was shifted in this case by $0.74 \mathrm{eV}$ (see Fig. 2(f)), which may have been due to the hydroxyl group $(-\mathrm{OH})$ being included in p-tert-butylcalix[4]arene molecular doping. Secondly, we found that the graphene induced peak disappeared. This phenomenon could be explained by the rehybridization of EG due to adsorbed hydroxyl group, which would break the mirror symmetry of the $\pi-\pi^{*}$ band crossing. Because we assigned the oxygen peak to the adsorption of hydroxyl group at the interface layer, the decrease in the density of states (DOS) at the Fermi level provided indirect evidence for the adsorption of hydroxyl groups on EG (Fig. 2(f)). Thirdly, the intensity of the peak at $4.7 \mathrm{eV}$ decreased with respect to the intensities of the other peaks as we compared valence band spectra shown in Fig. 2(e) with that shown in Fig. 2(f). This feature was the signature of the adsorption of hydroxyl group, and we assigned this band to the $\pi$ band of graphene at the M point.

We also measured the work function as a function of $p$-tertbutylcalix[4]arene exposure by monitoring the low kinetic ener-

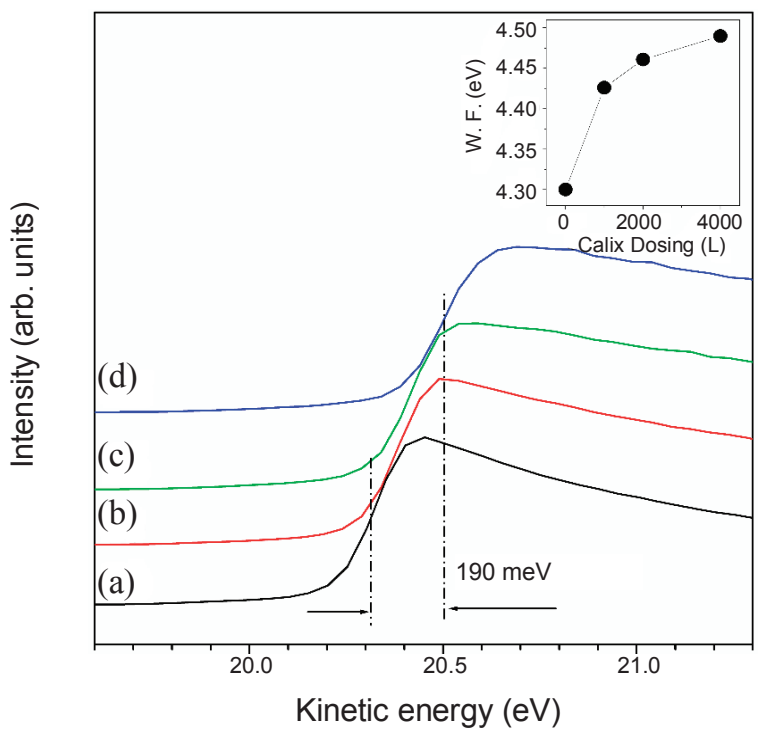

Figure 3. Work function measurements using the secondary electron cutoff with a photon energy of $80 \mathrm{eV}$ and a sample bias of $-20 \mathrm{~V}$. (a) Pristine monolayer graphene, (b) $500 \mathrm{~L}$, (c) $1000 \mathrm{~L}$, and (d) $4000 \mathrm{~L} p$ tert-butylcalix[4]arene adsorbed on EG. The inset shows the decrease in the work function as a function of $p$-tert-butylcalix[4]arene exposure. 


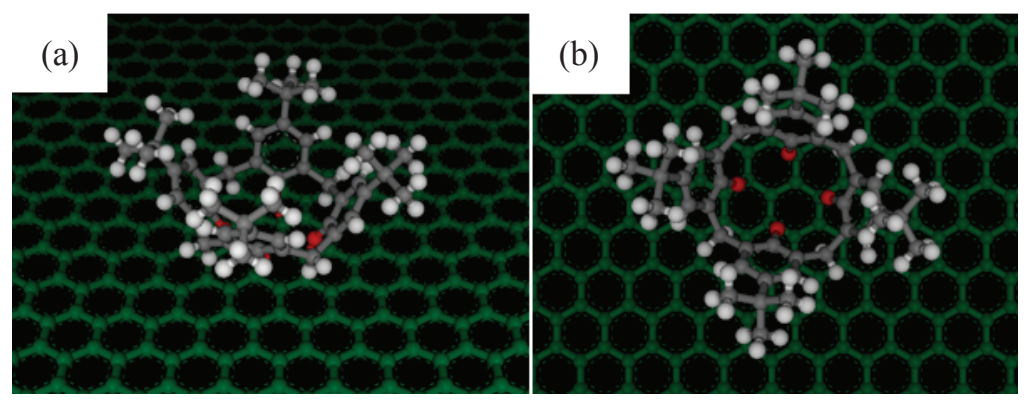

Figure 4. Schematic diagrams for $p$-tert-butylcalix[4]arene adsorbed on EG. (a) side view, (b) top view. Each red, gray, white, and dark cyan ball indicates oxygen, carbon, hydrogen, and graphene, respectively.

gy cutoff region, as shown in Fig. 3. Global doping effects on monolayer graphene were assessed by measuring the difference of the secondary electron edge between two systems, which is equal to the work function difference. ${ }^{25}$ The black spectrum displayed in Fig. 3(a) corresponds to the pristine monolayer graphene reference spectrum with $4.3 \mathrm{eV}$ work function value. ${ }^{26}$ As we increased the coverage of $p$-tert-butylcalix[4]arene up to $4000 \mathrm{~L}$ in Figs. 3(b) to 3(d), we clearly observed that the secondary electron edge shifted to higher kinetic energies by $190 \mathrm{meV}$ with respect to the pristine monolayer graphene. In other words, molecular doping by p-tert-butylcalix[4]arene increased the work function of graphene. The inset shown in Fig. 3 displays the increase in the work function of monolayer graphene, up to $4.49 \mathrm{eV}$, with increased doping of $p$-tert-butylcalix[4]arene. It is known that the sample work function of inert substrates such as graphite (excluding metal substrates) is extremely sensitive to interfacial charge transfer, and an upward shift of vacuum level or increase in sample work function occurs if electrons transfer from the substrate to the adsorbate overlayer. ${ }^{27-29}$ As proposed in Fig. 3, the strong electronaccepting characteristic of $p$-tert-butylcalix[4]arene favors electron transfer from EG into $p$-tert-butylcalix[4]arene, creating an interface dipole, which causes increase in sample work function indicating a typical $p$-type doping. Similar phenomena have been found in F4-TCNQ/EG systems, in which the increasing of the work function was also observed. ${ }^{6}$ Modifying the EG surface with p-tert-butylcalix[4]arene is therefore expected to favor electron transfer from EG into $p$-tert-butylcalix[4]arene molecules, leading to an electron accumulation layer in $p$-tert-butylcalix[4] arene and a depletion layer in EG, thereby achieving $p$-type doping of EG. As a result, we expect that the origin of $p$-type doping is induced by charge transfer from graphene to hydroxyl group being included in p-tertbutylcalix[4]arene due to the difference in electronegativity between carbon and oxygen.

Figure 4 displays the schematic diagram of the expected adsorption geometry of $p$-tert-butylcalix[4] arene adsorbed on EG. As shown in this model, the four oxygen in the p-tertbutylcalix[4] arene are expected to adsorb on the top sites of the graphene layer to form an $s p^{3}$ orbital, which is similar to the hydrogen on graphene system. ${ }^{30}$

\section{Conclusions}

We investigated the chemical doping of EG with p-tert- butylcalix[4]arene with a view to advancing doping technologies at the atomic scale beyond doping with hydroxyl group. HRPES was used to confirm the increase in the work function ( $p$-type). We investigated the variations in the electronic structure of hydroxyl group adsorbed on EG with increasing doses of $p$-tert-butylcalix[4]arene. We believe that the $p$-tert-butylcalix[4]arene-doped graphene layer may be useful for applications in electronic devices. The hydroxyl group in the $p$-tertbutylcalix[4] arene may possibly be utilized as an active center for the chemical functionalization of EG.

Acknowledgments. This work was supported by the Sookmyung Women's University Research Grants 2009.

\section{References}

1. Geim, A.; Novoselov, K. Nature Mater. 2007, 6, 183.

2. Ohta, T.; Bostwick, A.; Seyller, T.; Horn, K.; Rotenberg. E. Science 2006, 313, 951 .

3. Gierz, I.; Riedl, C.; Starke, U.; Ast, C. R.; Kern, K. Nano. Lett. 2008, 8, 4603 .

4. Bekyarova, E.; Itkis, M. E.; Ramesh, P.; Berger, C.; Sprinkle, M.; de Heer, W. A.; Haddon, R. C. J. Am. Chem. Soc. 2009, 131, 1336.

5. Zhou, S. Y.; Siegel, D. A.; Fedorov, A. V.; Lanzara, A. Phys. Rev. Lett. 2008, 101, 086402 .

6. Chen, W.; Chen, S.; Qi, D. C.; Gao, X. Y.; Wee, A. T. S. J. Am. Chem. Soc. 2007, 129, 10418.

7. Liu, L. H.; Yan, M. Nano Lett. 2009, 9, 3375.

8. Delamar, M.; Hitmi, R.; Pinson, J. ; Saveant, J. M. J. Am. Chem. Soc. 1992, 114, 5883.

9. Duplock, E. J.; Scheffler, M.; Lindan, P. J. D. Phys. Rev. Lett. 2004, 92, 225502.

10. Chernozatonski, L.; Sorokin, P.; Belova, E.; Brüning, J.; Fedorov, A. JETP Lett. 2007, 85, 77.

11. Pedersen, T. G.; Flindt, C.; Pedersen, J.; Mortensen, N. A.; Jauho, A.-P.; Pedersen, K. Phys. Rev. Lett. 2008, 100, 136804.

12. Giovannetti, G.; Khomyakov, P. A.; Brocks, G.; Kelly, P. J.; van den Brink, J. Phys. Rev. B 2007, 76, 073103.

13. Son, Y.; Cohen, M.; Louie, S. Phys. Rev. Lett. 2006, 97, 1.

14. Zhou, S. Y.; Siegel, D. A.; Fedorov, A. V.; El Gabaly, F.; Schmid, A. K.; Castro Neto, A. H.; Lee, D.-H.; Lanzara, A. Nature Mater. 2008, 7, 259.

15. Rotenberg, E.; Bostwick, A.; Ohta, T.; McChesney, J. L.; Seyller, T.; Horn, K. Nature Mater. 2008, 7, 258.

16. Krasheninnikov, A. V.; Banhart, F. Nature Mater. 2007, 6, 723.

17. Gutsche, C. D.; Iqbal, M. Organic Syntheses 1993, 8, 75.

18. Schreier, F. J. Quant. Spectrosc. Radiat. Transfer. 1992, 48, 743.

19. Kim, K.-j; Lee, H.; Choi, J.; Lee, H.-K.; Kang, T.-H.; Kim, B.; Kim, S. J. Phys.: Condens. Matter 2008, 20, 225017.

20. Tasis, D.; Tagmatarchis, N.; Bianco, A.; Prato, M. Chem. Rev. 2006, 
106, 1105-1136.

21. Holzinger, M.; Abraham, J.; Whelan, P.; Graupner, R.; Ley, L.; Hennrich, F.; Kappes, M.; Hirsch, A. J. Am. Chem. Soc. 2003, 125, 8566.

22. Johansson, L. I.; Owman, F.; Martensson, P. Phys. Rev. B 1996, $53,13793$.

23. Johansson, L. I.; Owman, F.; Martensson, P.; Persson, C.; Lindefelt, U. Phys. Rev. B 1996, 53, 13803.

24. Emtsev, K. V.; Speck, F.; Seyller, Th.; Ley, L.; Riley, J. D. Phys. Rev. $B$ 2008, 77, 155303

25. Cahen, D.; Kahn, A. Adv. Mater. 2003, 15, 271.

26. Alloway, D. M.; Hofmann, M.; Smith, D. L.; Gruhn, N. E.; Graham,
A. L.; Colorado, R., Jr.; Wysocki, V. H.; Lee, T. R.; Lee, P. A.; Armstrong, N. R. J. Phys. Chem. B 2003, 107, 11690.

27. Koch, N.; Duhm, S.; Rabe, J. P.; Vollmer, A.; Johnson, R. L. Phys. Rev. Lett. 2005, 95, 237601.

28. Koch, N.; Duhm, S.; Rabe, J. P.; Rentenberger, S.; Johnson, R. L.; Klankermayer, J.; Schreiber, F. Appl. Phys. Lett. 2005, 87, 101905.

29. Ishii, H.; Sugiyama, K.; Ito, E.; Seki, K. Adv. Mater. 1999, 11, 605 .

30. Balog, R.; Jørgensen, B.; Nilsson, L.; Andersen, M.; Rienks, E.; Bianchi, M.; Fanetti, M.; Lægsgaard, E.; Baraldi, A.; Lizzit, S.; Sljivancanin, Z.; Besenbacher, F.; Hammer, B.; Pedersen, T. G.; Hofmann, P.; Hornekær, L. Nature Materials 2010, 9, 315. 